\title{
Atenolol vs. propranolol for the treatment of infantile haemangiomas: A systematic review and meta-analysis
}

\author{
ZHUANG LIU, CHANGHUA WU, DAN SONG, LIANG WANG, \\ JING LI, CHANGFENG WANG and LEI GUO \\ Department of Vascular Anomalies and Interventional Radiology, \\ Qilu Children's Hospital of Shandong University, Jinan, Shandong 250022, P.R. China
}

Received September 19, 2019; Accepted April 1, 2020

DOI: $10.3892 /$ etm. 2020.8842

\begin{abstract}
Infantile haemangioma (IH) is a benign vascular tumour type that occurs in $3-10 \%$ of infants. In the present meta-analysis, previous studies comparing clinical outcomes, including the recovery rate and haemangioma activity score (HAS), adverse effects and relapse rates, were compared between patients treated with atenolol and those treated with propranolol for IH. A systematic search in various databases, including Medline, Cochrane Controlled Register of Trials, ScienceDirect and Google Scholar from inception until July 2019 was performed. The Cochrane risk of bias tool was used to assess the quality of published trials. A meta-analysis with a random-effects model and reported pooled mean differences (MD) or odds ratios (OR) with $95 \%$ CIs was performed. In total, 8 studies including 608 participants were analyzed. Only 2 studies were randomized controlled trials, while the majority of studies had low or unclear bias risks. Except for the response to medication (pooled $\mathrm{OR}=1.49 ; 95 \% \mathrm{CI}, 0.85-2.18$ ), all other outcomes (HAS, adverse reactions and relapse rate) were better for the atenolol group than the propranolol group. Atenolol resulted in better HAS (pooled $\mathrm{MD}=0.16$; 95\% CI, -0.42 to 0.73 ). Propranolol had more adverse reactions (pooled OR=2.17; 95\% CI, 0.93-5.06) and a higher relapse rate (pooled OR, 1.67; 95\% CI, 0.44-6.41) when compared to atenolol. However, these findings were not statistically significant. The results of this analysis suggest that atenolol may be non-inferior to propranolol and may offer advantages, including lower adverse reactions and relapse rates.
\end{abstract}

Correspondence to: Dr Lei Guo, Department of Vascular Anomalies and Interventional Radiology, Qilu Children's Hospital of Shandong University, 107 Wenhua West Road, Jinan, Shandong 250022, P.R. China

E-mail: 18866157186@163.com

Key words: atenolol, infantile haemangioma, meta-analysis, propranolol

\section{Introduction}

Infantile haemangioma (IH) is a type of benign vascular tumour that occurs in $3-10 \%$ of infants (1). While most of these lesions are asymptomatic and subside by the age of 5 years, complications may arise, including painful bleeding, ulceration or disfiguration $(2,3)$. IH may also induce mental distress to the parents and children (4). An early intervention is required in such cases to prevent future complications.

Systematic steroids were used as a first-line medication for the treatment of IH. However, the long-term use of steroids may lead to serious adverse reactions, including growth delay, adrenal cortical insufficiency and/or hypertension (5). To overcome these adverse reactions, The Food Drug Administration (FDA) of the US approved beta $(\beta)$ blockers as the first-line medications for the management of IH in 2014 (6). Propranolol is a non-selective lipophilic $\beta$ blocker proven to be effective against His (7) by inhibiting the proliferation and inducing the regression of the lesion during the proliferative phase (8). However, propranolol treatment also has certain risks, including side effects of diarrhoea, hyperkalaemia, hypoglycaemia and bronchial hyperreactivity. Propranolol also affects the central nervous system (CNS) as it crosses the blood-brain barrier due to its lipophilic nature and may cause adverse reactions, including agitation and sleep disturbances. These undesired effects from propranolol have led to discontinuation of therapy and regrowth of the lesions (9-11).

Atenolol, a hydrophilic $\beta$ blocker, has been used as an alternative to propranolol in the treatment of IH (12). Atenolol has minimal safety concerns, as it primarily acts on $\beta 1$ receptors with minor effects on $\beta 2$ receptors (13). As it does not act on pulmonary $\beta 2$ receptors, it may be safely used in infants with pulmonary diseases (e.g. reactive airway conditions). Atenolol also does not affect the pancreatic $\beta 2$ receptors and does not interfere with the glycogenolysis, gluconeogenesis or lipolysis (14). Due to its hydrophilic nature, it does not cross the blood-brain barrier and has limited adverse reactions when compared to propranolol (15). Studies have reported that atenolol is as effective as propranolol in reducing the size of the IH lesions $(13,14)$.

Even though the morbidity profile of atenolol for the management of IH has been established, only a few systematic studies have compared the clinical outcomes and/or adverse 
effects between these two different treatment methods $(16,17)$. The purpose of the present meta-analysis was to compare clinical outcomes [recovery rate, haemangioma activity score (HAS), adverse effects and relapse rates] between patients treated with atenolol and those treated with propranolol in the management of IH.

\section{Materials and methods}

Search strategy. An extensive search was performed in the following databases: Medline (PubMed) (https://pubmed. ncbi.nlm.nih.gov/), Google Scholar (https://scholar.google. com/), ScienceDirect (https://www.sciencedirect.com/) and Cochrane Central Register of Controlled Trials (CENTRAL) (https://www.cochranelibrary.com/central). In addition, a search was performed in the following clinical trial registries: ClinicalTrials.gov (https://clinicaltrials.gov/) and the World Health Organization International Clinical Trials Registry Platform (WHO ICTRP) (https://www.who. int/ictrp/search/en/). A combination of medical subject headings and free text terms, including 'haemangioma', 'atenolol', 'propranolol', 'beta blockers', 'adverse events', 'infants', 'infantile haemangioma', 'haemangioma activity score' and 'randomized controlled trial' were searched and all publications in English language from the database's inception to July 2019 were retrieved.

The reference lists of primary trials obtained through electronic searches were also checked and relevant articles were included for review and analysis. In cases requiring clarification or additional information, the authors of the published trials were contacted.

Inclusion and exclusion criteria. For inclusion in the present analysis, studies were required to fulfill all of the following criteria: i) Parallel arm individually randomized, quasi randomized and cluster randomized controlled trials (RCTs), and prospective/retrospective cohort studies, ii) studies on patients with $\mathrm{IH}$ and iii) studies comparing the effectiveness of atenolol and propranolol for IH management.

All cross-over studies were excluded due to the possibility of carryover effects. Only full-text/abstract publications were included.

Outcome measures. The following outcome measures were assessed: HAS, response to medication (reduction in the lesion size), adverse events and relapse rate. Studies reporting any of the above-mentioned outcomes and that met the inclusion criteria were included.

Selection of studies. The literature search was performed by two independent investigators (CW and DS) who screened the titles, abstracts and keywords of all the retrieved citations and assessed them for possible inclusion in the present analysis. Full-text articles of relevant studies were obtained and further screening was performed independently by the primary and secondary investigators (CW and DS) to select the studies satisfying the eligibility criteria for the present analysis. Any disagreements between investigators during the selection process were resolved either through consensus or consultation with another investigator (LW). A third investigator monitored the quality of the overall review process (LW). The Preferred Reporting Items for Systematic Review and Meta-Analysis checklist was used for reporting in this review (18).

Data extraction and management. The primary investigator $(\mathrm{CW})$ extracted the relevant study characteristics for review from the included studies and included general information, including the date of extraction, study title and authors; methods including the study design, participants and study setting; participant's characteristics, including the total number of participants in each arm, baseline and post-treatment outcome measures, and inclusion and exclusion criteria; intervention characteristics including details on the intervention and comparison group and follow-up duration; outcomes section, including primary and secondary outcomes, time taken for outcome assessment and other details necessary for assessing the risk of bias of included studies.

Primary and secondary investigators (CW and DS) independently extracted data associated with outcome measures from the studies included. Only extracted data from the relevant arms of studies reporting on multiple arms in a single trial were used for the present analyses. The primary investigator $(\mathrm{CW})$ transferred the obtained data to the statistical software RevMan 5.3 (Cochrane). The third investigator (LW) double-checked data entries for correctness by comparing them to the data in the studies.

Risk of bias assessment for the studies included. The risk of bias of included RCTs was assessed by two independent investigators (CW and DS) using the Cochrane risk of bias tool (19). The following domains were assessed: Random sequence generation, allocation concealment, blinding of outcome assessment and study participants, incomplete outcome data, selective reporting of outcomes and other sources of bias.

For non-randomized studies, the risk of bias assessment tool for non-randomized studies (20) was used with the following domains: Selection of participants, confounding variables, intervention measures, blinding of outcome assessment, incomplete outcome data and selective outcome reporting.

For each of the above-mentioned domains, the risk of bias was graded as low (if adequate information was provided), as high (if the information was inadequate or not performed) or as unclear (if the information was missing).

Statistical analysis. For continuous outcome (HAS), the mean and standard deviation (SD) reported at baseline and follow-up were obtained. In studies where change in mean and SD scores from baseline were reported, they were extracted directly. If change scores were not reported, manual calculation was performed using the following method:

Mean (change) was calculated as mean (after)-mean (before). Since the data were paired, equal variances were assumed for baseline and follow-up data. $n_{1}$ and $n_{2}$ were the number of participants at baseline and follow-up, respectively, while $s_{1}$ and $s_{2}$ were the standard deviations of baseline and follow-up, respectively.

The square of the SD was multiplied with the degrees of freedom: $\left(n_{1}-1\right) s_{1}^{2}$. This was repeated for the outcome: $\left(n_{2}-1\right) s_{2}{ }^{2}$. 
The two equations were added together and divided by the total degrees of freedom:

$$
s_{p}{ }^{2}=\frac{\left(n_{1}-1\right) s_{1}{ }^{2}+\left(n_{2}-1\right) s_{2}{ }^{2}}{n_{1}+n_{2}-2}
$$

The standard error (SE) of the difference between means was as follows:

$$
S E=\sqrt{\frac{s_{p}^{2}}{n_{1}}+\frac{s_{p}{ }^{2}}{n_{2}}}
$$

SD (change) was calculated with the equation: $\mathrm{SD}=\mathrm{SE} \times$ $\sqrt{ }$ (sample size). Mean (change) and SD (change) of both groups were then entered into meta-analysis software. Finally, pooled estimates were reported as Mean Difference (MD) with $95 \%$ CI.

For dichotomous variables including the response rate, adverse effects and relapse rate, the numbers of events and of participants in each group were obtained to estimate the pooled effect size in terms of odds ratios (most studies included were retrospective in nature).

Appropriate analyses were performed based on the level of randomization (either individual or clustered). No cluster randomized trials satisfying the eligibility criteria were identified and therefore, they did not require appropriate clustering adjustments. A random-effects model with inverse variance was utilized (21).

$\chi^{2}$ tests of heterogeneity to assess inter-study variance and $\mathrm{I}^{2}$ statistics were applied to quantify inconsistencies (19). Heterogeneity was classified according to $\mathrm{I}^{2}$ as mild $\left(\mathrm{I}^{2}<25 \%\right)$ moderate $\left(\mathrm{I}^{2}\right.$ between 25 and $\left.75 \%\right)$ or substantial $\left(\mathrm{I}^{2}>75 \%\right)$. Study-specific and pooled estimates were represented graphically through forest plots with random-effects model. None of the outcomes exhibited significant heterogeneity. Hence, subgroup analysis or meta-regression was not required for the present study.

Publication bias was not assessed, as the outcomes did not have the required number of studies (minimum of 10 studies) to assess the publication bias.

\section{Results}

Study selection. A systematic search to retrieve studies that directly compared the effectiveness of atenolol or propranolol for the management of $\mathrm{IH}$ from the dates of database inception until July 2019 was performed. A total of 798 citations, 383 studies retrieved from Medline, 134 from ScienceDirect, 177 from CENTRAL, 85 from Google Scholar, 12 from ClinicalTrials.gov and 7 from WHO ICTRP were identified (Fig. 1). After the first screening stage (title, abstract and keywords), 22 relevant studies were retrieved and their full texts were reviewed for eligibility. Simultaneously, the bibliographies of the studies retrieved were reviewed and 4 more relevant studies were identified. Finally, data from 8 studies with 608 participants satisfying the inclusion criteria were analysed (13,14,22-27).

Characteristics of studies included. Table I lists the characteristics of the studies analysed. Two studies were RCTs $(13,27)$, 3 were prospective $(23,25,26)$ and the remaining studies were retrospective studies $(14,22,24)$. Most of the studies were performed in Asian countries [China (2 studies) $(23,26)$ and India (1 study)] (27) and the others were performed in American and European countries. The mean age of the study participants ranged from 2 to 6.4 months in the atenolol group and that in the propranolol group ranged from 3 to 6 months. Of the 608 participants, 250 were in the atenolol cohort and 358 were in the propranolol group. The sample sizes in the studies (both groups together) ranged from 23 to 173, while the sample size in the atenolol group ranged from 7 to 82 patients and that in the propranolol group ranged from 10 to 98 . Among the 8 studies included, 6 reported on response to medication (reduction in the lesion size) $(13,22-26), 2$ reported on HAS $(14,27), 2$ reported on relapse rate $(13,25)$ and 6 reported on adverse effects following treatment $(13,14,23-25,27)$.

Methodological quality of the studies included. Assessments of risk of bias were performed separately for RCTs and non-randomized studies (Table IIA and B, respectively). There were no patients lost to follow-up reported in any of the studies included. The two RCTs $(13,27)$ included in the present meta-analysis had low risks of bias in almost all of the domains. Among the non-randomized studies (14,22-26), all of the studies had a low risk of bias regarding selection of participants, intervention measures, incomplete outcome data and unclear risks of selective reporting of outcomes (protocols not published), or blinding of outcome assessment (not mentioned in the studies). Furthermore, three out of six studies had high risks of bias with respect to confounding variables.

HAS. A total of two studies reported on HAS for the two groups (atenolol and propranolol) (14,27). Fig. 2 presents the pooled $\mathrm{MD}$ in the HAS at $0.16(95 \% \mathrm{CI},-0.42$ to 0.73$)$. This indicates that the evidence is not conclusive to determine which method results in a greater improvement in HAS. Furthermore, no significant heterogeneity was identified in the studies included reporting on $\operatorname{HAS}\left(\mathrm{I}^{2}=11 \%, \mathrm{P}=0.29\right)$.

Response to medication. Among the studies included, 6 reported on the response rate or reduction in the lesion size following intake of the medication in the two groups (atenolol and propranolol) (13,22-26). Apart from the study by Wang et al (26), all of the other studies $(13,14,22-25,27)$ indicated that propranolol was favoured, as it had a higher response rate when compared to the atenolol group. The overall pooled odds ratio (OR) in the propranolol arm was 1.36, indicating these infants had a 1.36 times greater odds of having complete response (reduction in lesion size) following the medication than those in the atenolol group (Fig. 3). However, the confidence of this pooled estimate crossed the null value of 1 (95\% CI, 0.85-2.18) and the result was not statistically significant. Furthermore, no heterogeneity among the studies reporting the response rate with $\mathrm{I}^{2}=0 \%$ was identified. The $\chi^{2}$ test for heterogeneity also indicated the absence of significant heterogeneity among the studies reporting on the response rate $(\mathrm{P}=0.43)$.

Adverse effects. A total of 6 studies reported on adverse effects following the medication in the 


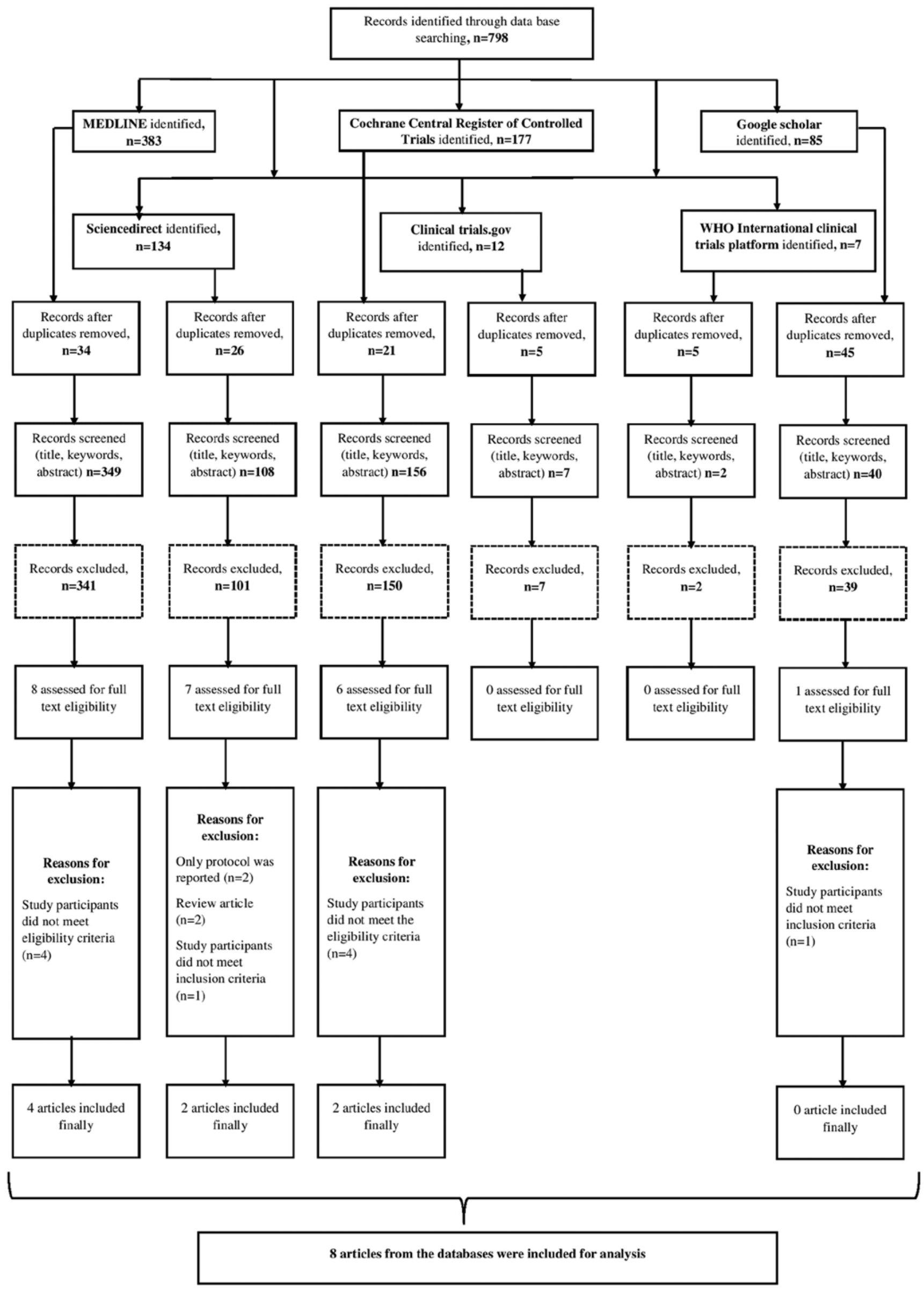

Figure 1. Preferred Reporting Items for Systematic Review and Meta-Analysis flow chart depicting the selection process of studies for the current review ( $\mathrm{n}=8$ ). WHO, World Health Organization. 


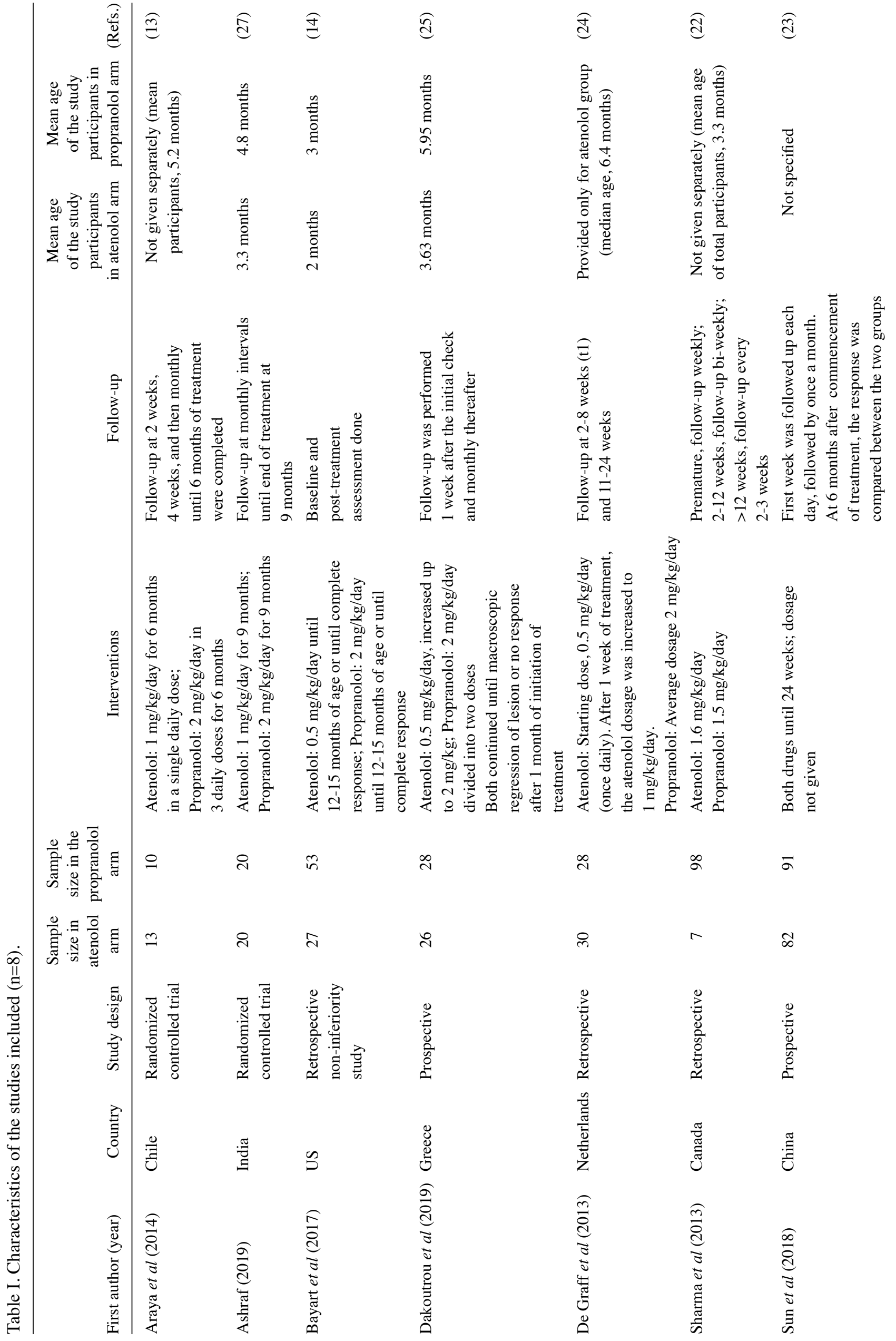


two groups $(13,14,23-25,27)$. Except for the studies by Ashraf et al (27) and Dakoutrou et al (25), all other studies reported that infants taking propranolol had a greater chance of developing adverse effects following medication when compared with infants taking atenolol. The overall pooled OR in the propranolol group was 2.17 , indicating these infants had a 2.17 times higher odds of developing adverse reactions following the medication than those in the atenolol group (Fig. 4). However, the confidence of this pooled estimate crossed the null value (95\% CI, 0.93-5.06) and the result was not statistically significant. Furthermore, moderate heterogeneity was present among the studies reporting on adverse effects with $\mathrm{I}^{2}=51 \%$. The $\chi^{2}$ test for heterogeneity indicated the absence of significant heterogeneity among the studies reporting on adverse effects $(\mathrm{P}=0.07)$.

Relapse rate. A total of 2 studies reported on the relapse rate following successful completion of treatment for the two groups (atenolol and propranolol) $(13,25)$. The overall pooled OR in the propranolol arm was 1.67 , indicating that these infants had a 1.67 times higher odds of relapse of IH following successful completion of medication than those in the atenolol group (Fig. 5). Similar to the above, the confidence of this pooled estimate crossed the null value (95\% CI, 0.44-6.41) and the result was not statistically significant. Furthermore, mild heterogeneity among the studies reporting on adverse effects with $\mathrm{I}^{2}=8 \%$ was obtained. The $\chi^{2}$ test for heterogeneity indicated an absence of significant heterogeneity among the studies reporting on adverse effects $(\mathrm{P}=0.45)$.

\section{Discussion}

The management of IH has varied historically and $\beta$ blockers have been the mainstay of treatment with a complete response rate of $\sim 60 \%$ (2). Each of the $\beta$ blockers used in the present analysis has its own advantages and disadvantages. There is a lack of systematic and high-quality studies assessing the effectiveness of these $\beta$ blockers directly. Hence, the present analysis was performed to compare the efficacies of atenolol and propranolol, in terms of clinical outcomes including the HAS and response rate, adverse effects and relapse rate among infants with haemangioma. The best possible evidence available to date was compiled in order to compare these medications.

A total of 8 studies comprising 608 participants were selected for inclusion in the present analysis. Of these, only 2 studies were RCTs and 3 were prospective studies, while the remainder were retrospective in nature. Most of the studies had either low or unclear bias risks. No substantial heterogeneity was identified among the reported outcomes in the studies. Hence, subgroup analysis or meta-regression was not performed to explore the source of heterogeneity. Except for the response to medication, all other outcomes (HAS, adverse reactions and relapse rate) were better for the atenolol group than the propranolol group. However, no conclusive or significant evidence for any of these outcomes was obtained, as the confidence limit crossed the null value in all of the outcomes assessed. The results from this analysis suggested 
Table II. Risk of bias assessment.

A, Randomized studies ( $\mathrm{n}=2)$

\begin{tabular}{|c|c|c|c|c|c|c|c|}
\hline Study & $\begin{array}{l}\text { Random } \\
\text { sequence } \\
\text { generation }\end{array}$ & $\begin{array}{c}\text { Allocation } \\
\text { concealment }\end{array}$ & $\begin{array}{c}\text { Blinding of the } \\
\text { participants, } \\
\text { outcome assessment }\end{array}$ & $\begin{array}{l}\text { Incomplete } \\
\text { outcome data }\end{array}$ & $\begin{array}{l}\text { Selective } \\
\text { reporting of } \\
\text { outcomes }\end{array}$ & $\begin{array}{l}\text { Other risk } \\
\text { of bias }\end{array}$ & (Refs.) \\
\hline Araya et al (2014) & Low risk & Low risk & Low risk & Low risk & Unclear risk & Low risk & (13) \\
\hline Ashraf et al (2019) & Low risk & Low risk & Low risk & Low risk & Unclear risk & Low risk & (27) \\
\hline
\end{tabular}

B, Non-randomized studies $(n=6)$

\begin{tabular}{|c|c|c|c|c|c|c|c|}
\hline Study & $\begin{array}{l}\text { Selection of } \\
\text { participants }\end{array}$ & $\begin{array}{c}\text { Confounding } \\
\text { variables }\end{array}$ & $\begin{array}{c}\text { Intervention } \\
\text { measures }\end{array}$ & $\begin{array}{l}\text { Blinding of } \\
\text { the outcome } \\
\text { assessment }\end{array}$ & $\begin{array}{l}\text { Incomplete } \\
\text { outcome } \\
\text { data }\end{array}$ & $\begin{array}{c}\text { Selective } \\
\text { reporting } \\
\text { of outcomes }\end{array}$ & (Refs.) \\
\hline Bayart et al (2017) & Low risk & High risk & Low risk & Unclear risk & Low risk & Unclear risk & (14) \\
\hline Dakoutrou et al (2019) & Low risk & Low risk & Low risk & Unclear risk & Low risk & Unclear risk & $(25)$ \\
\hline De Graff et al (2013) & Low risk & High risk & Low risk & Unclear risk & Low risk & Unclear risk & (24) \\
\hline Sharma et al (2013) & Low risk & High risk & Low risk & Unclear risk & Low risk & Unclear risk & $(22)$ \\
\hline Sun et al (2018) & Low risk & Low risk & Low risk & Unclear risk & Low risk & Unclear risk & (23) \\
\hline Wang et al (2016) & Low risk & Low risk & Low risk & Unclear risk & Low risk & Unclear risk & (26) \\
\hline
\end{tabular}

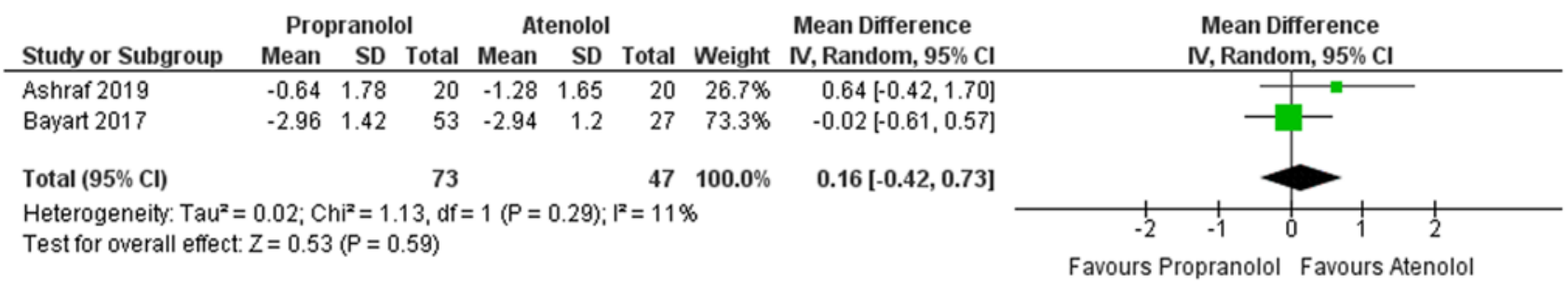

Figure 2. Forest plot indicating the difference in haemangioma activity score between the atenolol and propranolol arm $(\mathrm{n}=2)$. $\mathrm{SD}$, standard deviation; IV, inverse variance; df, degrees of freedom.

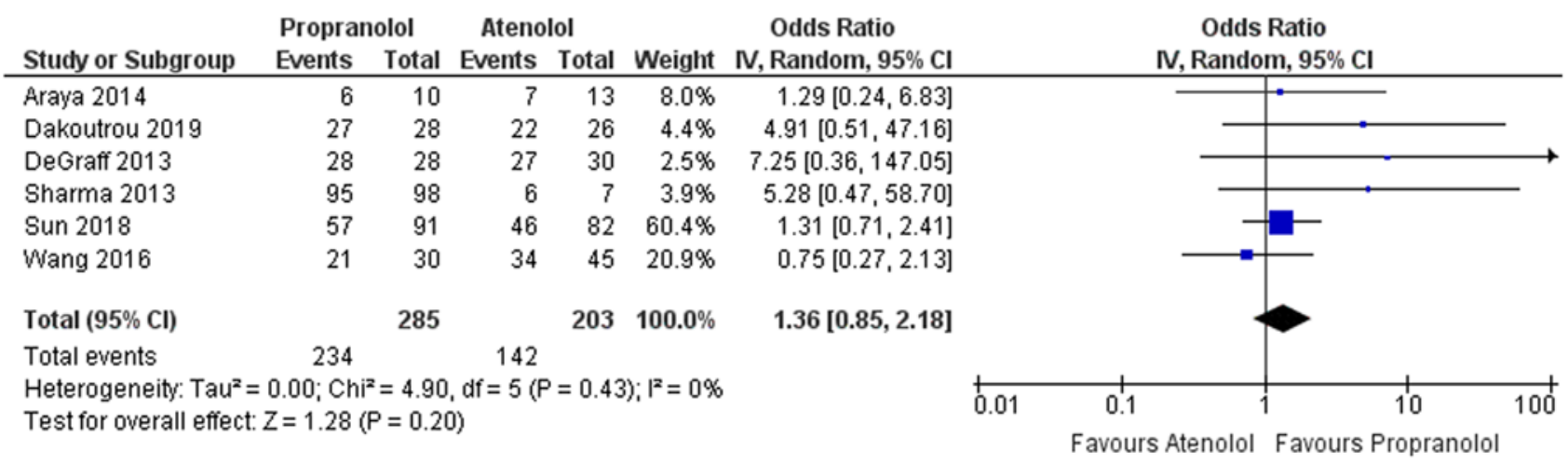

Figure 3. Forest plot analyzing the difference in response to medication between the atenolol and propranolol arm (n=6). SD, standard deviation; IV, inverse variance; $d f$, degrees of freedom.

that atenolol may be non-inferior to propranolol treatment in the management of IH. In almost all of the studies, atenolol was used at a dose of $0.5-1 \mathrm{mg} / \mathrm{kg} /$ day and propranolol at a dose of $2 \mathrm{mg} / \mathrm{kg} / \mathrm{day}$. However, the optimal dose of atenolol and propranolol remains to be determined, as there is a lack of dose-response studies.
The major strengths of the present study include the comprehensive literature search and the broad search strategy to include all of the relevant up-to-date publications. To the best of our knowledge, the present study was the first review directly comparing the clinical outcomes and adverse reaction profile between atenolol and propranolol for the management 


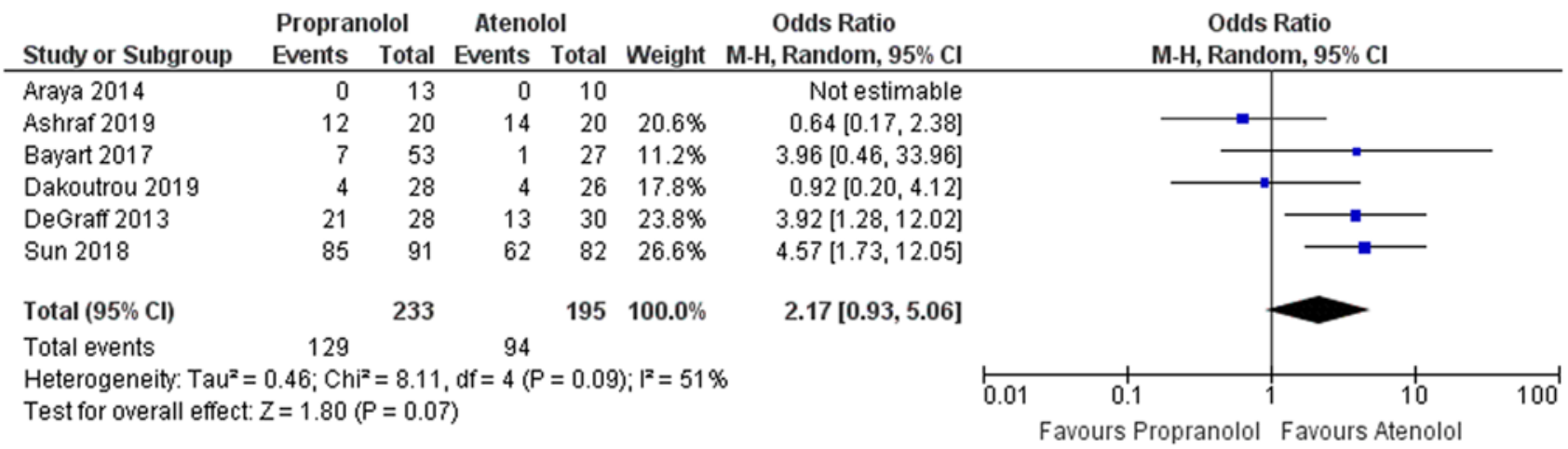

Figure 4. Forest plot for the difference in adverse reactions between atenolol and propranolol arm ( $\mathrm{n}=6)$. M-H, Mantel-Haenszel; df, degrees of freedom.

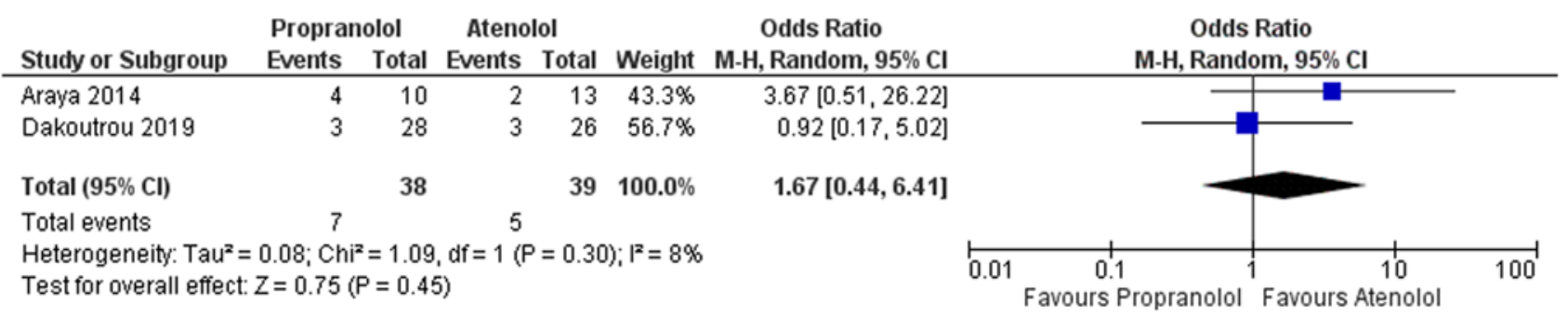

Figure 5. Forest plot indicating the difference in relapse rate between atenolol and propranolol arm ( $\mathrm{n}=2)$. M-H, Mantel-Haenszel; df, degrees of freedom.

of IH. Two previous reviews $(16,17)$ comparing propranolol with various other interventions have only included one study by Ábarzúa-Araya et al (13) to directly compare atenolol and propranolol.

The present review has certain limitations. Only 2 RCTs were included among the 8 studies. Since certain studies were retrospective in nature, no causal associations between the interventions and the outcomes can be inferred. Hence, more trials of adequate size are required to be performed to gather more evidence. It was not possible to assess publication bias, as the number of studies included in the review was $<10$ (minimum requirement to perform funnel plot or Egger's test) (19). There was insufficient information to determine the optimal dose for propranolol or atenolol, the optimal schedule or factors responsible for regrowth of $\mathrm{IH}$. Finally, most of the studies included in the present review were performed in high-income countries, which may limit the generalizability of the results to other geographical regions.

The results of the present study had certain implications towards clinical practice. Atenolol may be non-inferior to the propranolol treatment in the management of $\mathrm{IH}$. Propranolol is widely used as a first-line drug in the management of complications. Previous evidence has indicated that propranolol has potential adverse effects on the development of the CNS of infants. It is known to negatively influence psychomotor function or the memory of infants. In addition, bronchial-associated adverse effects (e.g. bronchial hypersensitivity) have been reported propranolol users. With the current evidence, clinicians are able to use atenolol in place of propranolol depending on the patient's profile (i.e. if the infants require medication having a minimal effect on bronchus or CNS) or it may be used as an alternative if the infant on propranolol develops side effects. However, uncertainties regarding the efficacy and safety persist, as most of the studies have an inadequate sample size that limits the power of the studies.

Apart from efficacy and safety concerns, questions regarding the dose-response association to determine the optimal dose, schedule and factors responsible for regrowth or relapse of $\mathrm{IH}$ following treatment require further exploration. To obtain conclusive evidence on these factors, more RCTs or prospective studies with larger sample sizes are required to strengthen the evidence for recommendations on how to best treat infants with haemangioma, as $\beta$ blockers are the only FDA-approved drug for this condition.

To summarize, atenolol may be non-inferior to propranolol in the management of IH with respect to clinical outcomes and adverse reactions. However, more RCTs with larger sample sizes are required to derive conclusive evidence towards efficacy, safety and dose-response association of atenolol and propranolol.

\section{Acknowledgements}

Not applicable.

\section{Funding}

No funding was received.

\section{Availability of data and materials}

The datasets used and/or analyzed during the current study are available from the corresponding author on reasonable request. 


\section{Authors' contributions}

ZL designed the study. C Wu, DS, LW, JL, C Wang and LG were involved in literature search and data interpretation. $\mathrm{C}$ $\mathrm{Wu}$ and DS were responsible for the data analysis. ZL prepared the manuscript. LG edited the manuscript. All authors read and approved the final manuscript.

\section{Ethics approval and consent to participate}

Not applicable.

\section{Patient consent for publication}

Not applicable.

\section{Competing interests}

The authors declare that they have no competing interests.

\section{References}

1. Frieden IJ, Haggstrom AN, Drolet BA, Mancini AJ, Friedlander SF, Boon L, Chamlin SL, Baselga E, Garzon MC Nopper AJ, et al: Infantile hemangiomas: Current knowledge, future directions. Proceedings of a research workshop on infantile hemangiomas, April 7-9, 2005, Bethesda, Maryland, USA. Pediatr Dermatol 22: 383-406, 2005.

2. Darrow DH, Greene AK, Mancini AJ and Nopper AJ; Section On Dermatology, Section On Otolaryngology-Head And Neck Surgery, and Section On Plastic Surgery: Diagnosis and management of infantile hemangioma. Pediatrics 136 e1060-1104, 2015.

3. Drolet BA, Frommelt PC, Chamlin SL, Haggstrom A, Bauman NM, Chiu YE, Chun RH, Garzon MC, Holland KE, Liberman L, et al: Initiation and use of propranolol for infantile hemangioma: Report of a consensus conference. Pediatrics 131: 128-140, 2013.

4. Csoma ZR, Dalmády S, Ábrahám R, Rózsa T, Rácz K and Kemény L: Infantile haemangioma: Clinical and demographic characteristics, experiences in the treatment. Orv Hetil 158 : 1535-1544, 2017 (In Hungarian).

5. Maturo S and Hartnick C: Initial experience using propranolol as the sole treatment for infantile airway hemangiomas. Int J Pediatr Otorhinolaryngol 74: 323-325, 2010.

6. Léauté-Labrèze C, Hoeger P, Mazereeuw-Hautier J, Guibaud L, Baselga E, Posiunas G, Phillips RJ, Caceres H Lopez Gutierrez JC, Ballona R, et al: A randomized, controlled trial of oral propranolol in infantile hemangioma. N Engl $\mathrm{J}$ Med 372: 735-746, 2015.

7. Léaute-Labrèze $\mathrm{C}$, Boccara $\mathrm{O}$, Degrugillier-Chopinet $\mathrm{C}$, Mazereeuw-Hautier J, Prey S, Lebbé G, Gautier S, Ortis V, Lafon M, Montagne A, et al: Safety of oral propranolol for the treatment of infantile hemangioma: A systematic review. Pediatrics 138 e20160353,2016.

8. Zhang L, Wu HW, Yuan W and Zheng JW: Propranolol therapy for infantile hemangioma: Our experience. Drug Des Devel Ther 11: 1401-1408, 2017.

9. Raphael MF, Breur JM, Vlasveld FA, Elbert NJ, Liem YT, Kon M, Breugem CC and Pasmans SG: Treatment of infantile hemangiomas: Therapeutic options in regard to side effects and adverse events-a review of the literature. Expert Opin Drug Saf 15: 199-214, 2016

10. Léauté-Labrèze C, Harper JI and Hoeger PH: Infantile haemangioma. Lancet 390: 85-94, 2017.
11. Chang L, Gu Y, Yu Z, Ying H, Qiu Y, Ma G, Chen H, Jin Y and Lin X: When to stop propranolol for infantile hemangioma. Sci Rep 7: 43292, 2017.

12. Raphaël MF, de Graaf M, Breugem CC, Pasmans SGMA and Breur JMPJ: Atenolol: A promising alternative to propranolol for the treatment of hemangiomas. J Am Acad Dermatol 65: 420-421, 2011.

13. Ábarzúa-Araya A, Navarrete-Dechent CP, Heusser F, Retamal J and Zegpi-Trueba MS: Atenolol versus propranolol for the treatment of infantile hemangiomas: A randomized controlled study. J Am Acad Dermatol 70: 1045-1049, 2014.

14. Bayart CB, Tamburro JE, Vidimos AT, Wang L and Golden AB: Atenolol versus propranolol for treatment of infantile hemangiomas during the proliferative phase: A retrospective noninferiority study. Pediatr Dermatol 34: 413-421, 2017.

15. Wang Q, Xiang B, Chen S and Ji Y: Efficacy and safety of oral atenolol for the treatment of infantile haemangioma: A systematic review. Australas J Dermatol 60: 181-185, 2019.

16. Liu X, Qu X, Zheng J and Zhang L: Effectiveness and safety of oral propranolol versus other treatments for infantile hemangiomas: A meta-analysis. PLoS One 10: e0138100, 2015.

17. Novoa M, Baselga E, Beltran S, Giraldo L, Shahbaz A, Pardo-Hernandez $\mathrm{H}$ and Arevalo-Rodriguez I: Interventions for infantile haemangiomas of the skin. Cochrane Database Syst Rev 4: CD006545, 2018.

18. Liberati A, Altman DG, Tetzlaff J, Mulrow C, Gøtzsche PC, Ioannidis JP, Clarke M, Devereaux PJ, Kleijnen J and Moher D: The PRISMA statement for reporting systematic reviews and meta-analyses of studies that evaluate health care interventions: Explanation and elaboration. J Clin Epidemiol 62: e1-e34, 2009.

19. Higgins JP and Green S (eds.): Cochrane Handbook for Systematic Reviews of Interventions. John Wiley \& Sons, Ltd., Chichester, 2008

20. Kim SY, Park JE, Lee YJ, Seo HJ, Sheen SS, Hahn S, Jang BH and Son HJ: Testing a tool for assessing the risk of bias for nonrandomized studies showed moderate reliability and promising validity. J Clin Epidemiol 66: 408-414, 2013.

21. Rao G, Lopez-Jimenez F, Boyd J, D'Amico F, Durant NH, Hlatky MA, Howard G, Kirley K, Masi C, Powell-Wiley TM, et al: Methodological standards for meta-analyses and qualitative systematic reviews of cardiac prevention and treatment studies: A scientific statement from the American heart association. Circulation 136: e172-e194, 2017.

22. Sharma VK, Fraulin FO, Dumestre DO, Walker L and Harrop AR: Beta-blockers for the treatment of problematic hemangiomas. Can J Plast Surg 21: 23-28, 2013.

23. Sun L, Sun B, Ma Y et al: Comparison of the efficacy and safety of propranolol and atenolol in the treatment of infant hemangioma. Progress Modern Biomed 18: 108-112, 2018.

24. de Graaf M, Raphael MF, Breugem CC, Knol MJ, Bruijnzeel-Koomen CA, Kon M, Breur JM and Pasmans SG: Treatment of infantile haemangiomas with atenolol: Comparison with a historical propranolol group. J Plast Reconstr Aesthet Surg 66: 1732-1740, 2013

25. Dakoutrou M, Alexopoulos A, Miligkos M, Georgiadou E, Kanaka-Gantenbein C and Kakourou T: Atenolol treatment for severe infantile hemangiomas: Comparison with a propranolol group of our centre. J Eur Acad Dermatol Venereol 33: e199-e200, 2019.

26. Wang Q, Xiang B, Ji Y, Li F, Xu Z and Zhong L: Propranolol versus atenolol in the treatment of infantile hemangioma: A comparative study. Chin J Dermatol 49: 683-687, 2016.

27. Ashraf R, Mahajan R, Abas M, Handa S, Sinha A, De D and Sachdev N: Comparing the clinical, radiologic and biochemical effectiveness of atenolol and propranolol in the treatment of infantile hemangioma-a randomized controlled trial. Social Science Research Network, Rochester, NY, 2019. https://ssrn. com/abstract $=3353380$.

This work is licensed under a Creative Commons Attribution-NonCommercial-NoDerivatives 4.0 International (CC BY-NC-ND 4.0) License. 